\title{
Understanding Body MRI Sequences and Their Ability to Characterize Tissues
}

\author{
Anuj Patel $^{1, *}$, Chad Silverberg ${ }^{2}$, David Becker-Weidman ${ }^{1},{\text { Christopher } \text { Roth }^{1} \text {, Sandeep Deshmukh }}^{1}$ \\ ${ }^{1}$ Department of Radiology, Thomas Jefferson University, Philadelphia, USA \\ ${ }^{2}$ Progressive Radiology, Greenbelt, USA
}

Copyright (C) 2016 by authors, all rights reserved. Authors agree that this article remains permanently open access under the terms of the Creative Commons Attribution License 4.0 International License

\begin{abstract}
Familiarity with how MRI sequences can distinguish different tissues when coupled with an understanding of pathology aids in narrowing differentials or making a specific diagnosis. Utilizing specific MRI pulse sequences allows for identification of key tissue substances such as fat, paramagnetic substances, protein, fibrous tissue, or free or bound water. The identification of these tissue substances allows the radiologist to form narrow or specific diagnoses efficiently. A tissue-based approach to understanding MRI sequences allows the radiologist to both systematically and effectively interpret MRIs despite the large number of pulse sequences particularly in basic MRI body protocols.
\end{abstract}

Keywords MRI Sequences, Tissue Characteristics, Body MRI

\section{Introduction}

A fundamental strength of MRI lies in the ability to create tissue contrast based upon the histologic composition of both physiologic and pathologic entities. As the modality has advanced, a litany of sequences has been developed and continues to further this concept. However, the sheer number of sequences can be daunting to both the trainee and seasoned MR imagers and may lead to overlooking of subtle findings which are only depicted on certain sequences. Additionally, the naming convention for different MRI sequences varies with each of the different vendors which creates further diagnostic hurdles in imaging interpretation. Radiologists often work in practices which utilize multiple MRI vendors and are expected to be able to interpret the images independent of the naming convention of the sequences. Therefore, identifying the strengths and weaknesses of depicting different tissue types on each sequence of a basic MRI protocol allows for systematic and effective image interpretation.
Table 1. Summary of Imaging Characteristics Based on Tissue Type

\begin{tabular}{|c|c|}
\hline TISSUE & IMAGING FEATURES \\
\hline $\begin{array}{l}\text { Paramagnetic } \\
\text { Substances (iron, } \\
\text { calcium, metal) }\end{array}$ & $\begin{array}{c}\text { Decreased signal on In Phase relative to Out of } \\
\text { Phase }\end{array}$ \\
\hline Microscopic Fat & $\begin{array}{c}\text { Decreased signal on Out of Phase relative to In } \\
\text { Phase } \\
\text { Macroscopic fat with not show signal loss on In } \\
\text { Phase or Out of Phase }\end{array}$ \\
\hline Macroscopic Fat & $\begin{array}{l}\text { Signal nulling via Inversion Recovery or } \\
\text { Frequency Selective techniques. } \\
\text { Inversion Recovery has more uniform fat signal } \\
\text { suppression. }\end{array}$ \\
\hline Hemorrhage & $\begin{array}{l}\text { Complex signal characteristics depending on age of } \\
\text { blood products } \\
\text { Subacute blood products are } \mathrm{T} 1 \text { hyperintense and } \\
\text { T2 hypointense/hyperintense } \\
\text { Chronic blood products are } \mathrm{T} 1 \text { and } \mathrm{T} 2 \text { hypointense }\end{array}$ \\
\hline Melanin & Most commonly T1 hyperintense \\
\hline Protein & $\begin{array}{c}\text { Intermediate or hyperintense } \mathrm{T} 1 \text { signal } \\
\text { Pancrease is brightest organ in the body on } \mathrm{T} 1 \\
\text { weighted image }\end{array}$ \\
\hline Fibrosis & $\begin{array}{l}\mathrm{T} 1 \text { and } \mathrm{T} 2 \text { hypointese } \\
\text { Delayed enhancement }\end{array}$ \\
\hline Solid Tissue & Early enhancement \\
\hline $\begin{array}{l}\text { Free Water } \\
\text { (Cysts) }\end{array}$ & $\begin{array}{l}\text { Markedly hyperintense on moderately and heavily } \\
\text { T2 weighted images } \\
\text { Signal loss in mesenteric/retroperitoneal fat on Out } \\
\text { of Phase compared to In Phase imaging }\end{array}$ \\
\hline $\begin{array}{l}\text { Bound Water } \\
\text { (cancer or benign } \\
\text { lesions) }\end{array}$ & $\begin{array}{l}\text { Mildly hyperintense on moderately T2 weighted } \\
\text { image } \\
\text { No signal are mild signal on heavily T2 weighted } \\
\text { image } \\
\text { Concern for malignancy in liver if near isointense } \\
\text { to spleen on moderately T2 weighted image } \\
\text { Typically benign if hyperintense to spleen on } \\
\text { moderately T2 weighted image }\end{array}$ \\
\hline
\end{tabular}




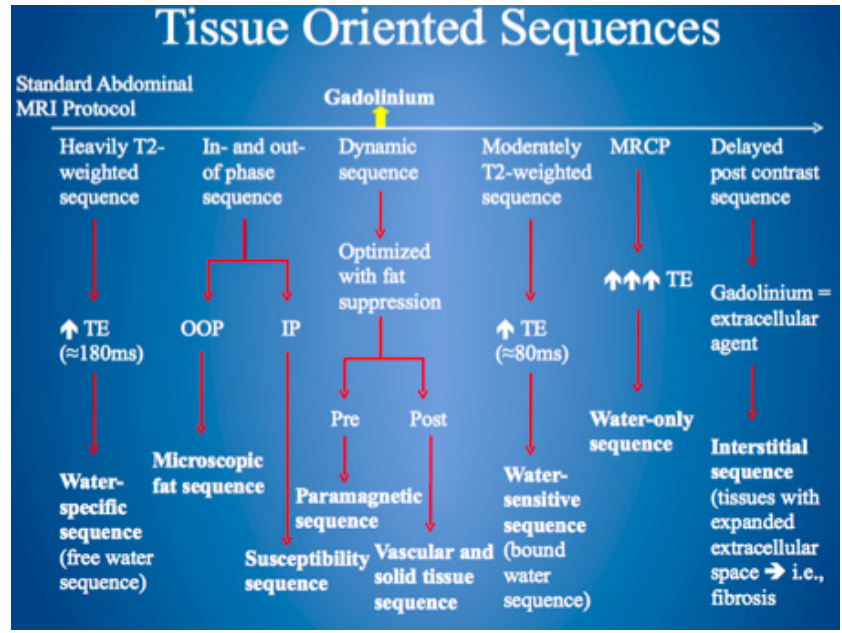

Figure 1. Standard abdominal MRI protocol including sequences with high sensitivity for free and bound water, microscopic and macroscopic fat, paramagnetic substances, fibrosis, and solid tissue.

Abdominal MRI pulse sequences are individually designed to distinguish specific tissues. Complemented with a fundamental histologic understanding of common physiologic and disease states, creation of tissue contrast based upon the presence or absence of tissue types can allow for a narrow differential diagnosis or a specific diagnosis. A classification scheme that separates sequences into a tissue specific approach serves as a manageable means to fully utilize the advantages of individual sequences in a typical MRI protocol (Table 1 and Figure 1).

Evaluation of pathologic states based on the presence or absence of tissue types can be readily categorized if the same methodical approach is used to interpret each study. An understanding of how different tissue types appear on each of the basic MRI sequences facilitates a high yield, fast, reproducible, and accurate interpretation of abdominal MRI exams.

\section{Microscopic Fat}

Conventional diagnostic MRI measures signal from hydrogen ions in a given volume. Although the most prominent hydrogen containing molecule in the human body is water, hydrogen ions in other molecules also contribute signal and therefore to tissue contrast [1]. Hydrogen-containing molecules most frequently contributing to signal other than water include proteins, carbohydrates, and fats.

The primary sequence directed toward separating signal of hydrogen ions in water from those of fats is referred to as chemical shift or in and out of phase imaging. This consists of a gradient echo sequence at a specific TE, in order to take advantage of the principle that water and fat alternate between precession at identical or complete opposite phases when excited by a specific frequency. When water and fat are contained in the same voxel, finite time intervals exist in which hydrogen ions in fat versus those in water either precess in phase and contribute to signal, or precess out of phase by 180 degrees, leading to nulling of signal. In effect, in phase images have contribution from both fat and water hydrogen ions to create signal while out of phase images yield signal cancellation, leading to absence of signal [2,3].

Many pathologic entities in abdominal imaging contain fat, which can increase the sensitivity and specificity for diagnosis upon identification. Selected examples of several common fat containing lesions include adrenal adenomas, hepatocellular carcinoma, and hepatic steatosis. Identification of fat in one of these lesions significantly narrows the differential diagnosis, and can often be diagnostic (Figure 2). Within the liver, the presence of microscopic fat in a lesion is useful in identifying the lesion of hepatocellular in origin such as hepatocellular carcinoma, hepatic adenoma, or hepatic steatosis. Cysts, hemangioma, and most metastases do not contain lipid with few rare exceptions [4].

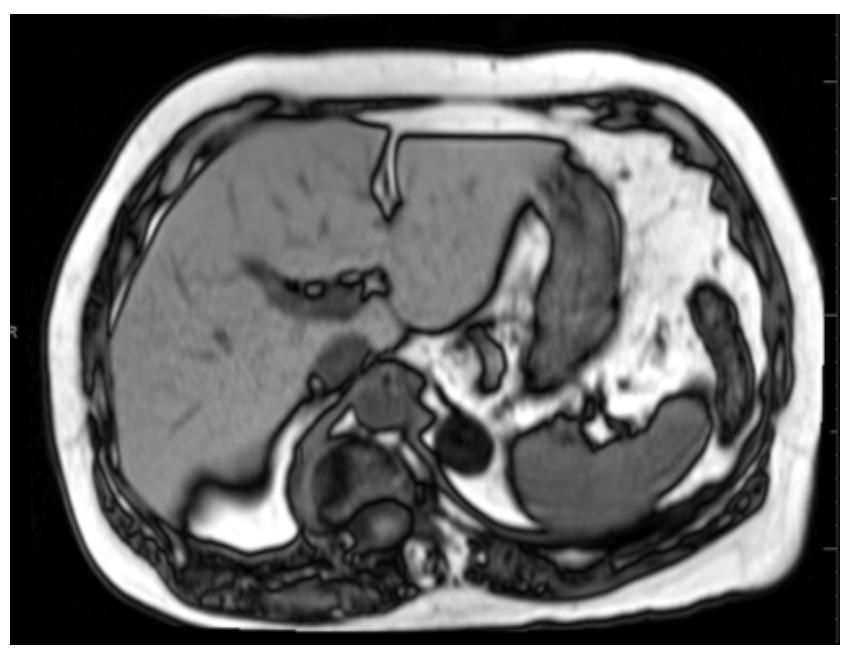

A

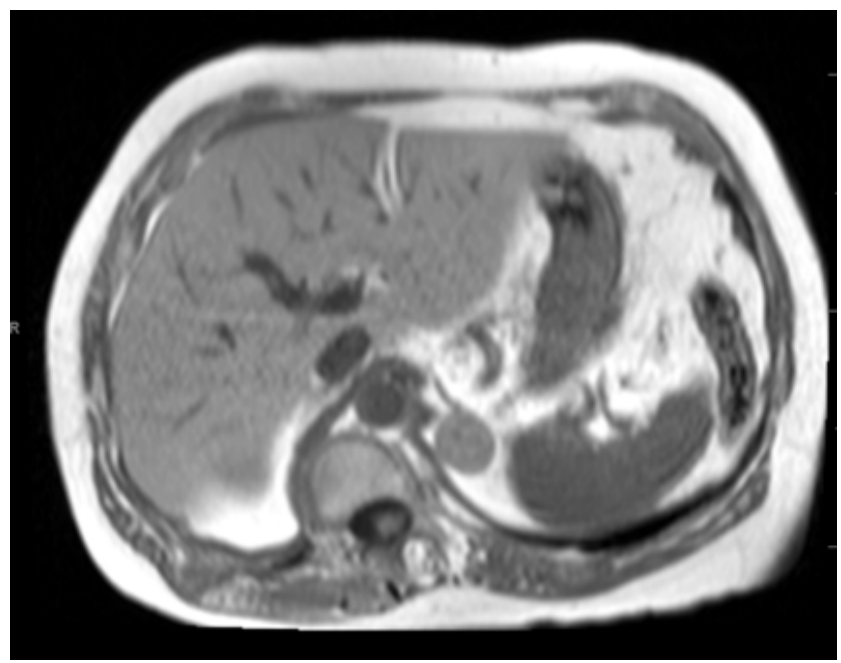

B

Figure 2. Out of phase (A) and In phase (B) gradient echo sequences demonstrating loss of signal in a left adrenal mass, diagnostic for an adrenal adenoma. Signal loss in Out of phase image within the left adrenal mass is due to fat and water within the same imaged voxel.

Chemical shift imaging is most useful for microscopic fat, 
which is more likely to contain water in the same voxel when compared to macroscopic fat. This can be seen when evaluating subcutaneous fat. Subcutaneous fat contains little to no water and thus does not null, therefore contributing to signal on the out of phase sequences.

In order to achieve in and out phase sequences, two distinct TEs corresponding to water and fat in phase and out of phase are selected. As TE increases, susceptibility artifact created by paramagnetic substances is also increased, adding an additional function of this sequence. Paramagnetic ions, most often iron or calcium, become more apparent as TE increases (blooming), and can be more easily identified when compared to spin echo sequences (Figure 3) [5].

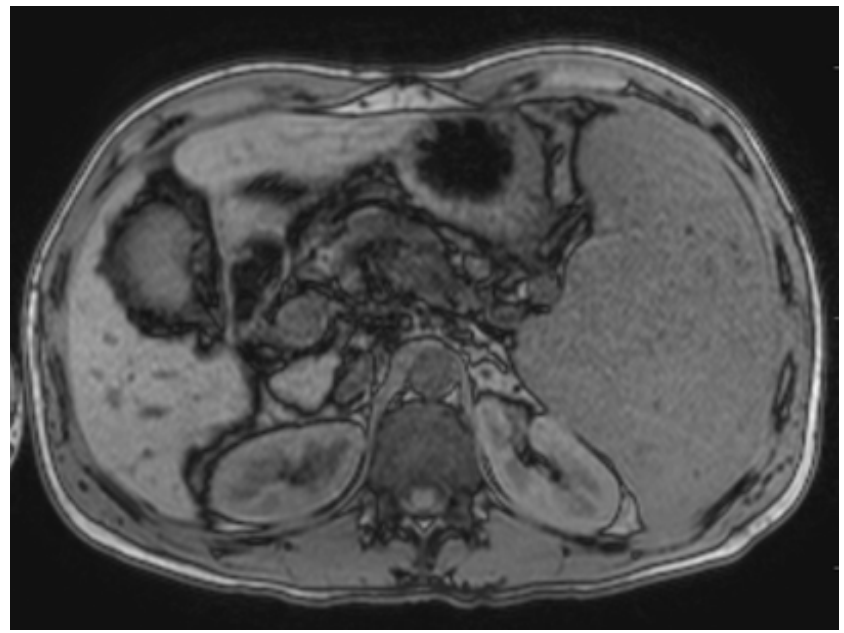

A

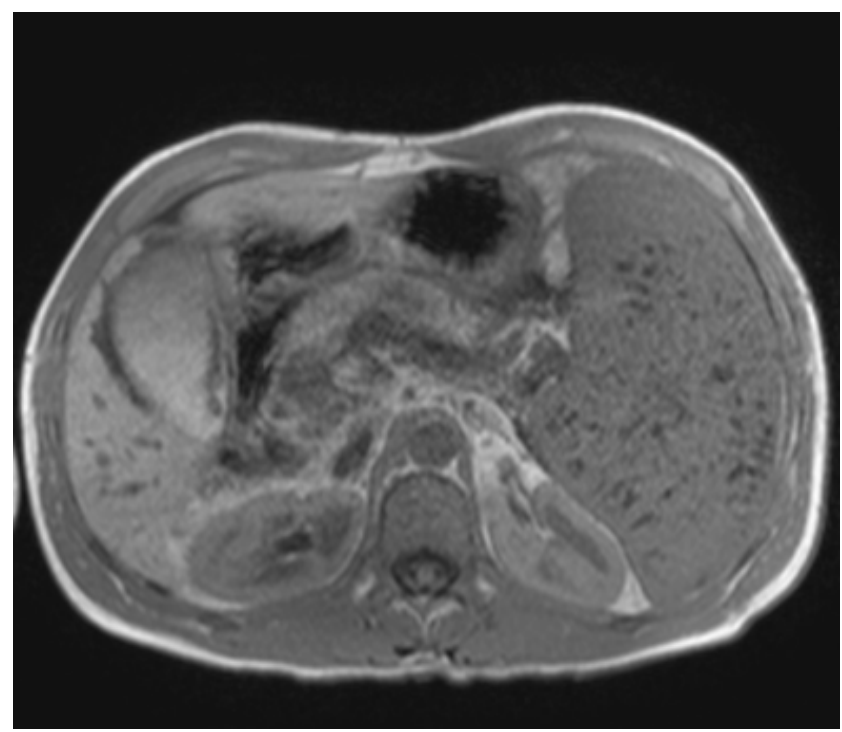

B

Figure 3. Out of phase (A) and in phase (B) gradient echo sequences demonstrating punctate foci of susceptibility artifact (blooming) within the spleen in a patient with splenomegaly and cirrhosis. This is secondary to iron deposition in the spleen (Gamna Gandy bodies). Note that as TE increases from the out of phase to in phase sequence, susceptibility artifact increases. The principle of increased susceptibility artifact on In phase sequences only occurs if the In phase sequence has a longer TE compared to the corresponding Out of phase sequence.

In phase imaging must always be obtained at a longer TE than out of phase. If the out of phase sequence is obtained with a longer TE than in phase, one would not be able to assess if signal loss on the longer TE sequence is due to microscopic fat or susceptibility [6].

\section{Macroscopic Fat}

Fat is consistently bright on conventional pulse sequences, often leading to decreased tissue contrast between fat and T1/ T2 bright pathology. Fat suppression is employed to null the signal from fat, and thus render contribution of non-fat containing bright T1/T2 signal more apparent. Several methods have been utilized to accomplish this task, including inversion recovery and frequency selective fat suppression. Inversion recovery often results in more homogenous fat suppression; however, both techniques improve tissue contrast [7].

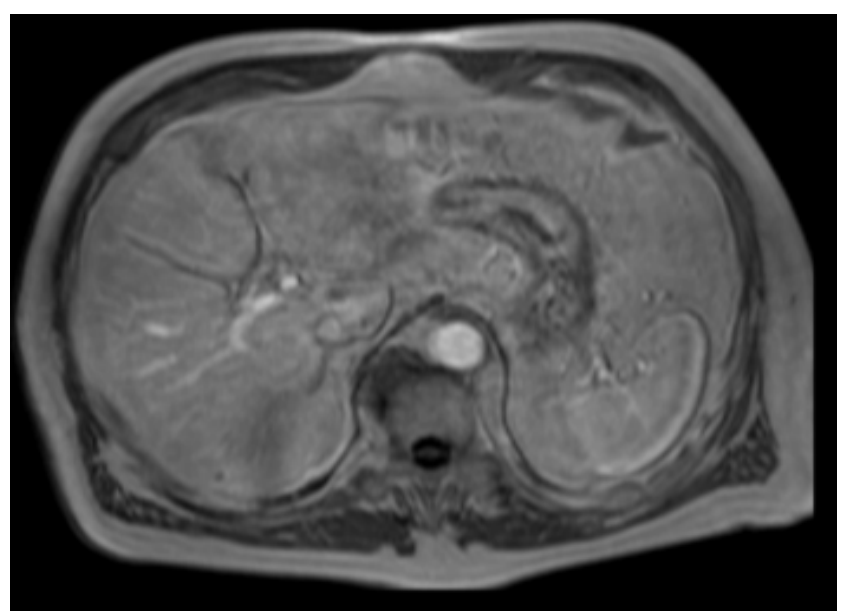

A

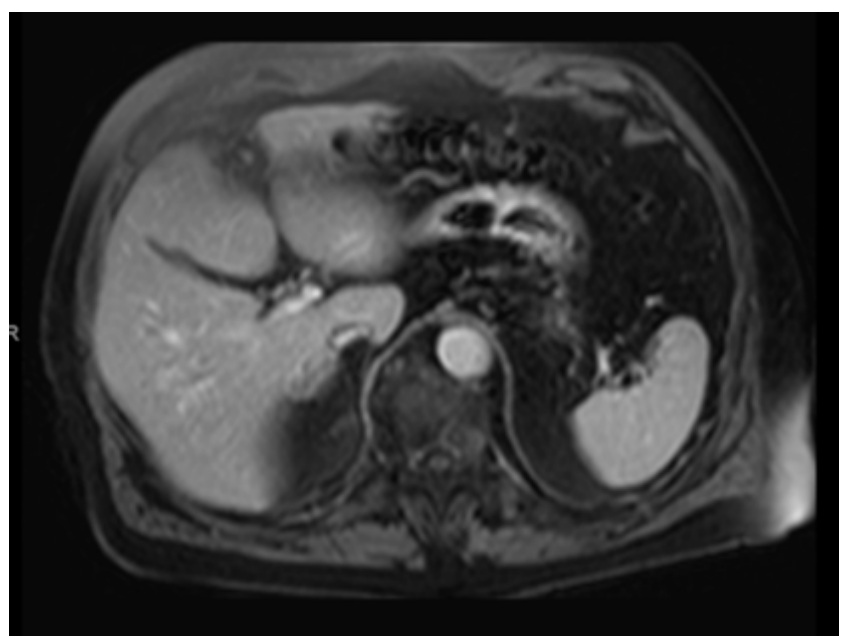

B

Figure 4. Post gadolinium gradient echo sequence without fat suppression (A) and with fat suppression (B). Note the increased conspicuity of the hemorrhagic pancreatitis secondary to increased dynamic range. Fat suppression is not possible in Figure 4A due to lower magnet field strength. Fat suppression is unreliable with low field magnets because the difference in precessional frequency between fat and water is proportional to field strength. At lower field strengths, it is difficult to have frequency-selective saturation pulses specific to fat. 
Fat suppression using these methods is most useful for macroscopic fat, such as suppressing subcutaneous or intra-abdominal fat to increase the dynamic range of the sequence, as is employed in post gadolinium sequences (Figure 4). Multiple pathologic states, however also contain macroscopic fat, such as renal angiomyolipomas and adrenal myelolipomas (Figure 5). Identification of macroscopic fat in these lesions can narrow the differential diagnosis.
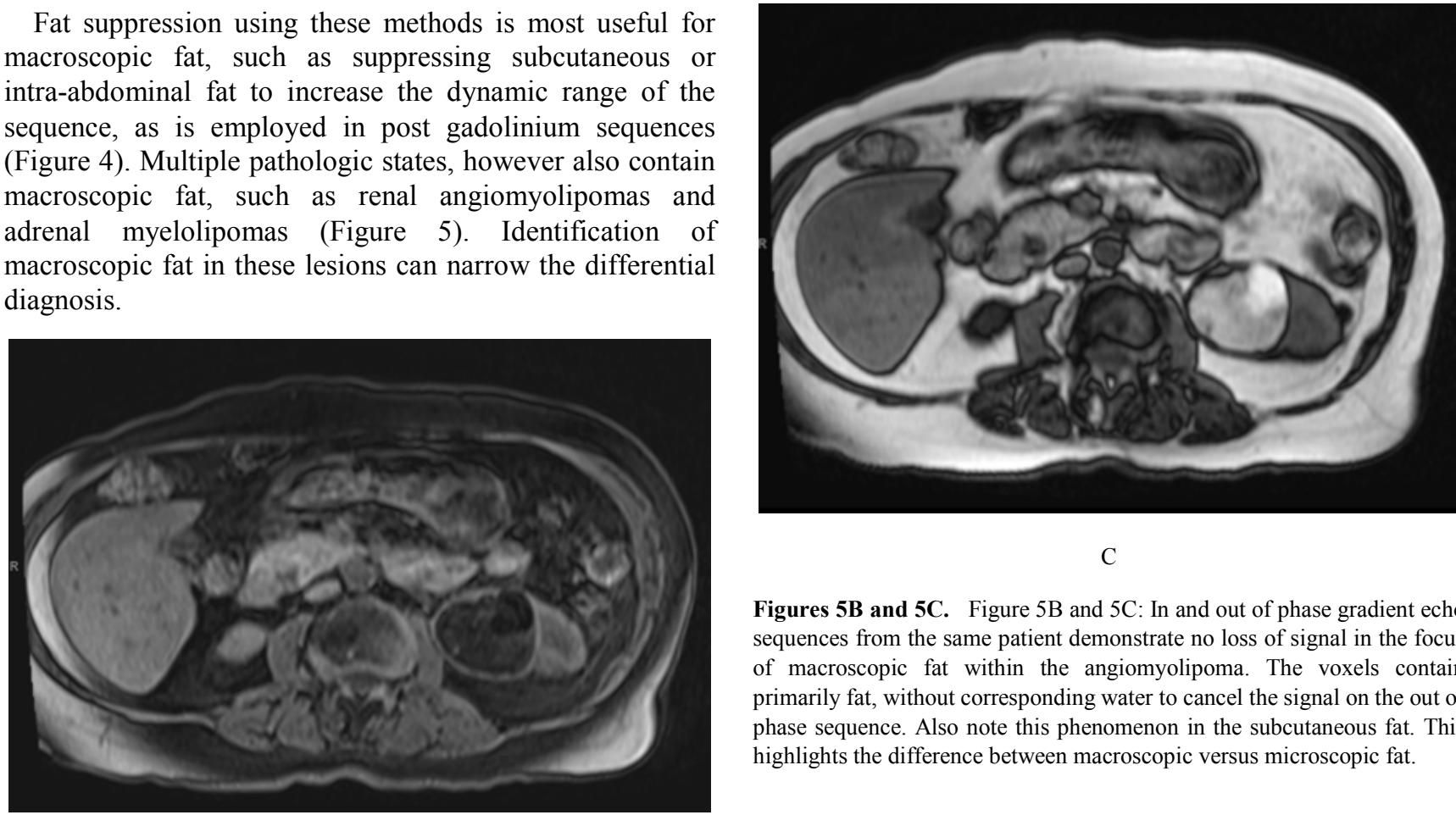

C

Figures 5B and 5C. Figure 5B and 5C: In and out of phase gradient echo sequences from the same patient demonstrate no loss of signal in the focus of macroscopic fat within the angiomyolipoma. The voxels contain primarily fat, without corresponding water to cancel the signal on the out of phase sequence. Also note this phenomenon in the subcutaneous fat. This highlights the difference between macroscopic versus microscopic fat.

\section{Hemorrhage}

demonstrating an exophytic left renal mass. A small focus of hypointense T1 signal is noted in the anterior aspect of the mass, similar in signal to the suppressed subcutaneous fat. This is consistent with macroscopic fat within an angiomyolipoma.

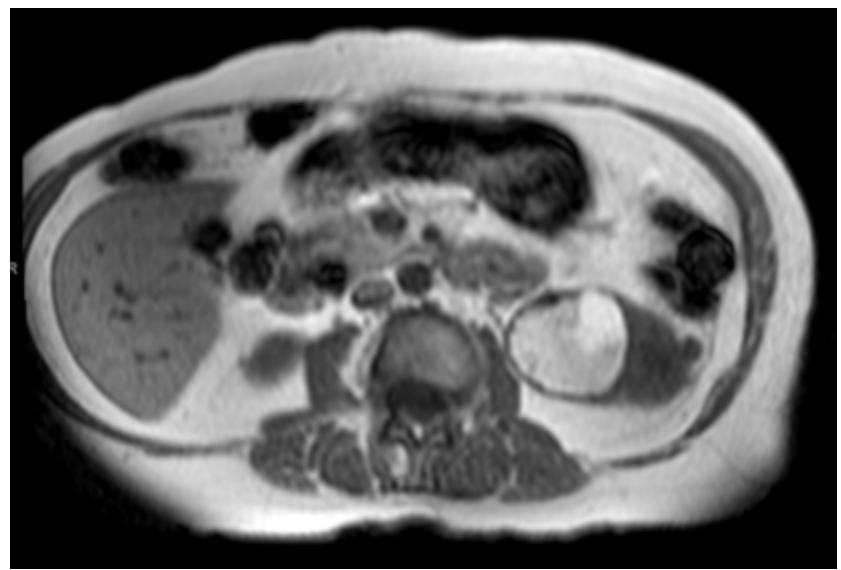

Hemoglobin contains a heme moiety and proteinaceous globular components, which can lead to a complex MR appearance when pathologically located outside of red blood cells. As hemoglobin degrades, the globin molecules are dissociated from the heme moiety, and the iron molecule undergoes oxidation. This occurs in stages depending upon several factors, including intra versus extracellular location of hemoglobin, degradation state of the globin molecule, and oxidation state of the heme moiety. Characteristic MRI appearance of these stages can allow for diagnosis of chronicity of blood products. This occurs in a more predictable fashion in compartments with high oxygen tension, such as the brain, and most hemorrhage in abdominal imaging is identified in the subacute or chronic stage, however the same general principles apply [8]. The most common stage of hemorrhage in abdominal MRI is subacute, which demonstrates $\mathrm{T} 1$ hyperintensity and $\mathrm{T} 2$ hypo or hyperintensity (Figure 6). 


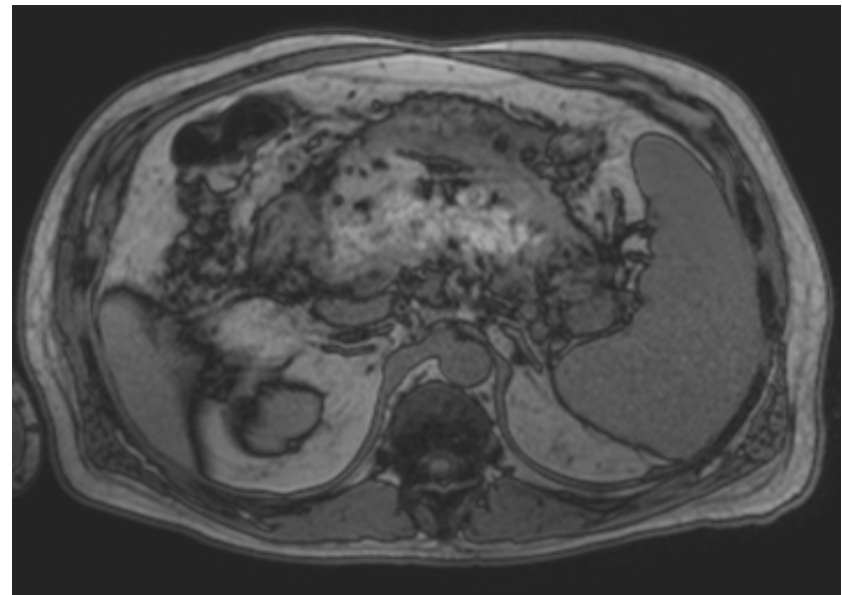

Figure 6. Out of phase gradient echo $\mathrm{T} 1$ weighted sequence demonstrates hyperintense $\mathrm{T} 1$ signal in the bed of the pancreas, with obscuration of the surrounding peripancreatic fat planes. This corresponds to subacute blood products in a patient with hemorrhagic pancreatitis.

Hemorrhage can be associated with pathologic entities, both traumatic and non-traumatic. MRI is often utilized to further evaluate indeterminate renal lesions identified on CT or ultrasound. An important distinction of renal masses is that of a hemorrhagic cyst versus neoplasm. Identification of hemorrhage in the absence of enhancing tissues can be diagnostic for this entity (Figure 7).

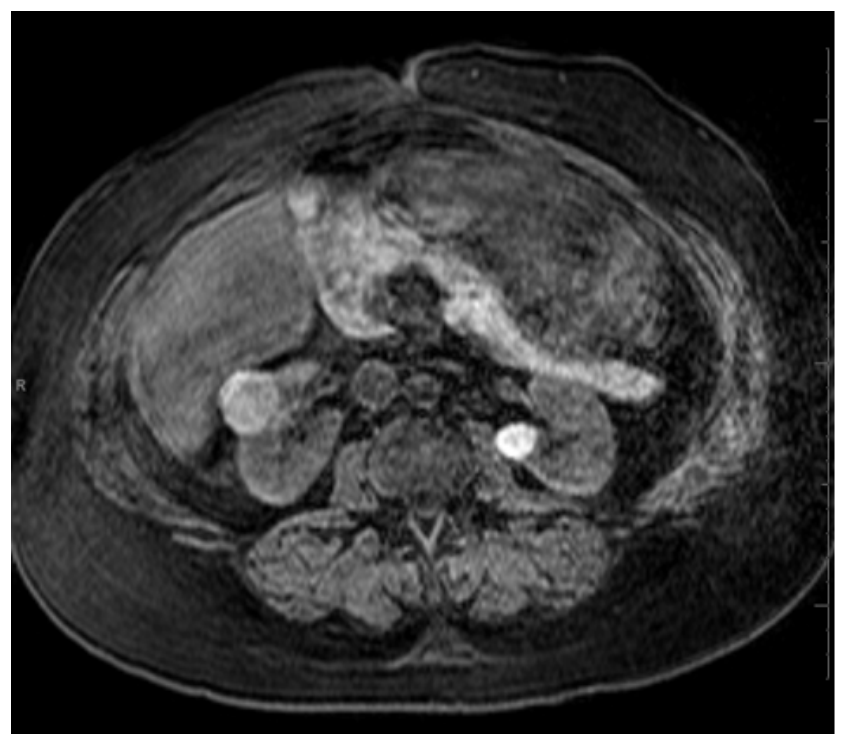

Figure 7. Fat saturated pre-gadolinium $\mathrm{T} 1$ weighted $3 \mathrm{D}$ gradient echo sequence showing bilateral $\mathrm{T} 1$ hyperintense renal lesions, representing hemorrhagic renal cysts containing subacute blood products.

\section{Melanin}

Melanoma is a malignant neoplasm of melanocytes that has unique imaging properties, allowing for high diagnostic sensitivity in abdominal MRI. Melanin is thought to bind to paramagnetic substances that cause T1 shortening, leading to a typical T1 bright appearance [9]. It should be noted that not all melanoma lesions are T1 bright, and metastatic melanoma can also appear as $\mathrm{T} 1$ hypointense. When paramagnetic substances are present in melanin, identification can significantly shorten the differential diagnostic considerations. For example, uveal melanoma has a tendency to metastasize to liver, and the typical T1 bright nature of this lesion can aid in screening patients (Figure 8).

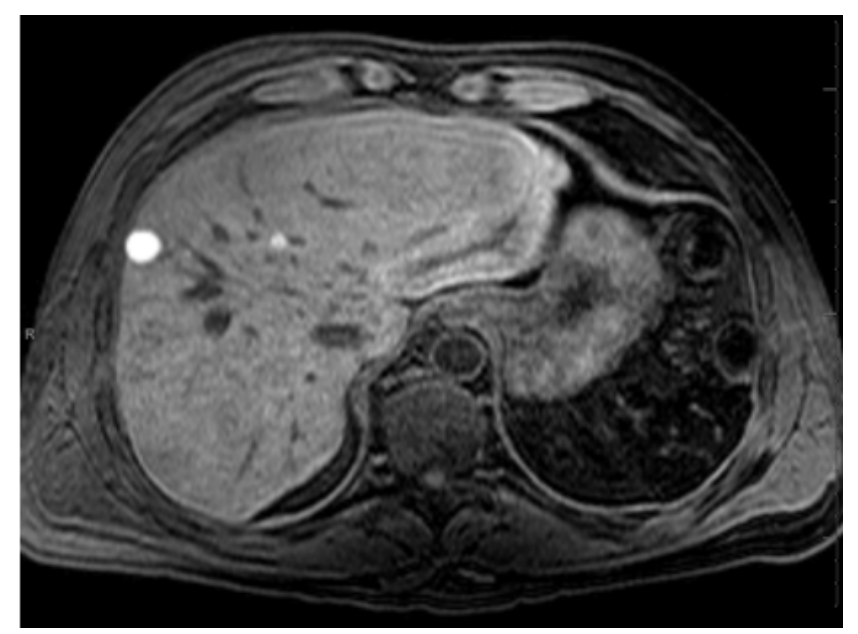

Figure 8. Fat saturated pre-gadolinium $\mathrm{T} 1$ weighted $3 \mathrm{D}$ gradient echo sequence demonstrating two hyperintense $\mathrm{T} 1$ lesions in the right hepatic lobe. This patient had known uveal melanoma, and these represented melanotic metastases.

Melanotic metastases are typically hypervascular and have a propensity to spread to organs less commonly affected by non-melanoma metastatic disease. Once a melanotic metastasis is identified, a judicious search for lesions in atypical locations is warranted.

\section{Protein}

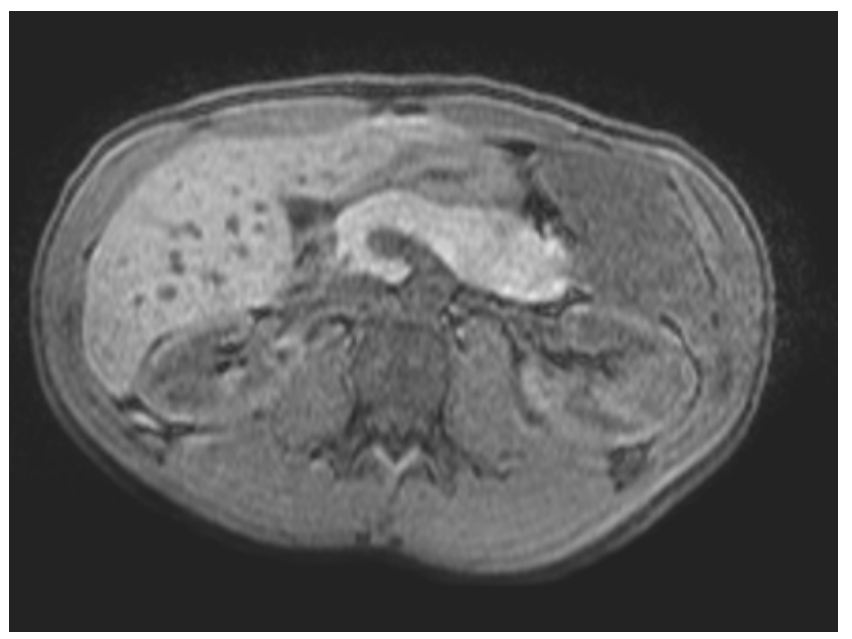

Figure 9. Fat saturated Pre-gadolinium T1 weighted 3D gradient echo sequence demonstrating normal $\mathrm{T} 1$ hyperintense pancreatic parenchyma in a healthy male adult. Note the pancreas is the brightest organ in the abdomen.

Identification of protein can be indicative of both physiologic and pathologic states, and is typically T1 bright. The pancreas produces protein hormones, including insulin 
in significant amounts, which likely contributes to the pancreas being the brightest abdominal organ on $\mathrm{T} 1$ weighted images [10] (Figure 9). This can be helpful in a normal checklist when reviewing abdominal MRI cases. Lack of this relationship on T1 weighted sequences can be indicative of pathology such as pancreatitis or underlying mass. The lack diffuse or focal lack of relative T1 hyperintensity can often be the only salient abnormality.

Protein can also help to differentiate benign from malignant processes, such identifying proteinaceous contents of a cyst which was indeterminate as an echogenic mass on ultrasound or hyperdense cyst on CT. MR can offer a unifying diagnosis, obviating the need for further evaluation or intervention.

\section{Fibrous Tissue}

Fibrous tissues has a typical imaging appearance in abdominal MRI of low signal on both T1 and T2 weighted sequences, as well as enhancement on delayed phase post gadolinium T1 weighted sequences. Collagen deposition is a common reparative process that takes place in injured parenchyma. Once intravenous contrast has reached the interstitial phase, in parenchyma that has undergone injury, contrast is able to extend into spaces previously occupied by cells, which leads to greater enhancement of fibrotic areas relative to background parenchyma. This characteristic appearance can help to offer a specific diagnosis in entities in which fibrosis is suspected or expected.

Cirrhosis and chronic pancreatitis are disease states, which often demonstrate parenchymal fibrosis as the sequela of chronic injury [11] (Figure 10). This can also be seen in tumors that are predominantly composed of fibrous material, such as desmoid tumors (Figure 11).

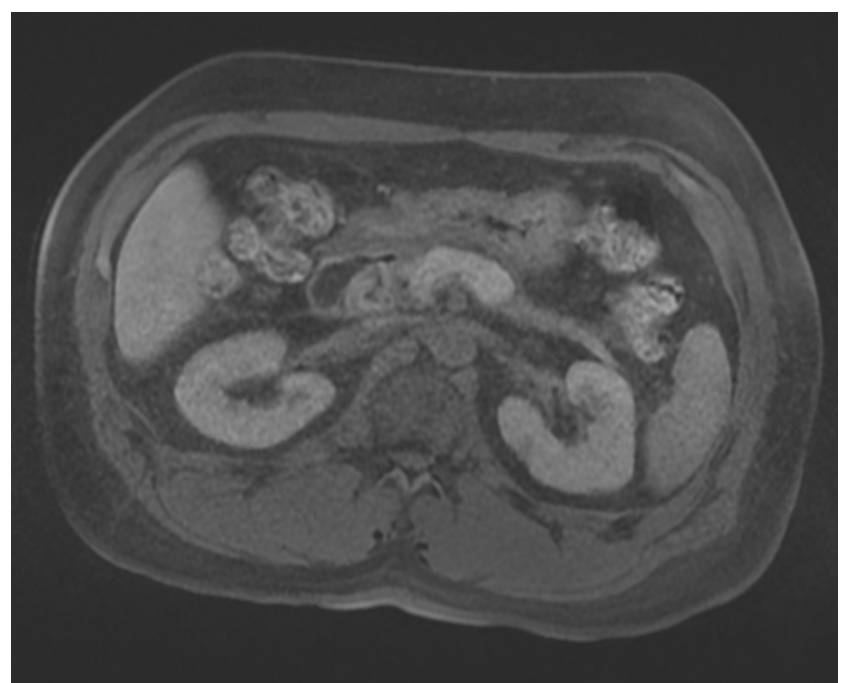

Figure 10. Fat saturated pre-gadolinium $\mathrm{T} 1$ weighted $3 \mathrm{D}$ gradient echo sequence demonstrating low T1 signal in the pancreatic tail, corresponding to parenchymal fibrosis in a patient with chronic pancreatitis. Normal pancreatic parenchyma is typically the most $\mathrm{T} 1$ hyperintense organ in the abdomen.

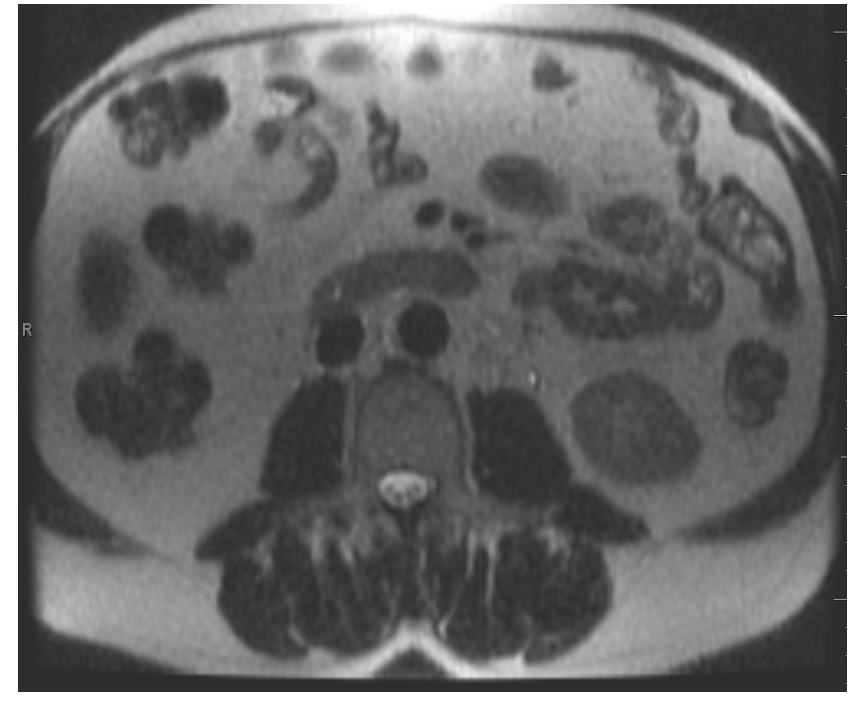

A

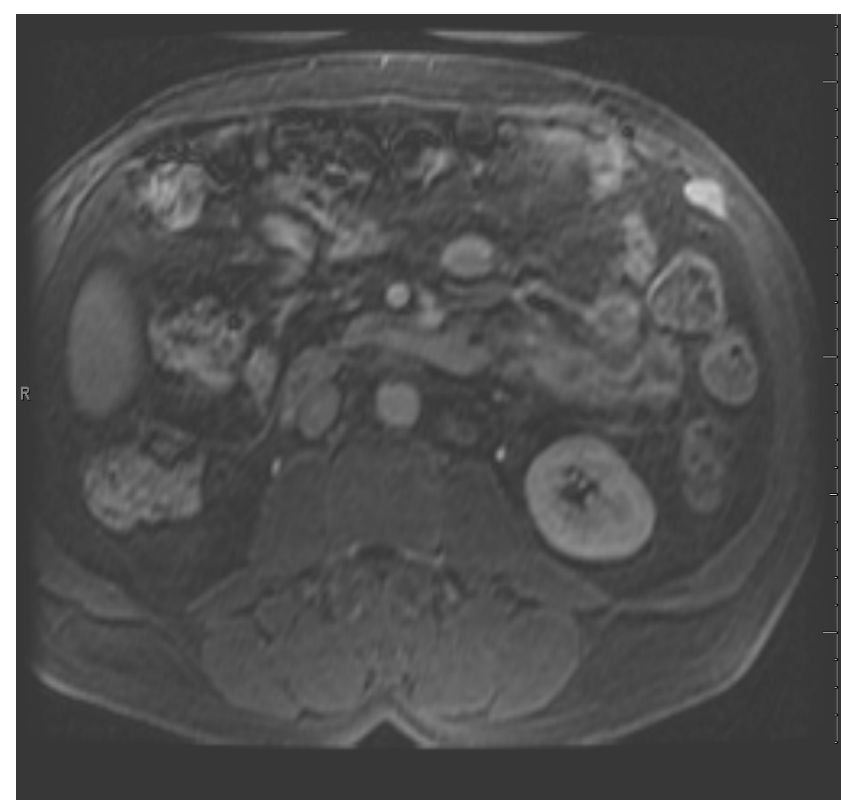

Figure 11. Axial T2 (A) and axial fat saturated post-gadolinium (B) sequences demonstrate a T2 hypointense, enhancing left ventral abdominal wall desmoid tumor. Low T2 signal corresponds to the fibrous nature of the tumor and presence of enhancement signifies the solid nature of the mass.

\section{Solid Tissue}

Determination of the cystic versus solid nature of a lesion can drastically changes the significance and management of many lesions found in abdominal MRI. Solid lesions usually have a vascular supply, denoting viable tissue. Identification of this property can be accomplished by administration of intravenous gadolinium and subsequent demonstration of enhancement.

Neoplastic processes frequently have the ability for angiogenesis, thereby taking up gadolinium relative to non-vascular tissues. As gadolinium shortens T1, enhancing 
tissues appear bright on T1 weighted images relative to background. The blood vessels formed by tumor angiogenesis are immature, and while avidly siphon contrast from larger central vessels during the arterial phase of a dynamic sequence, often leak contrast leading to rapid washout [12].

Hepatocellular carcinoma is a vascular tumor that classically demonstrates enhancement on post gadolinium $\mathrm{T} 1$ weighted sequences. In patient populations at risk for development of HCC, screening exams should ideally include gadolinium-enhanced images (Figure 12).

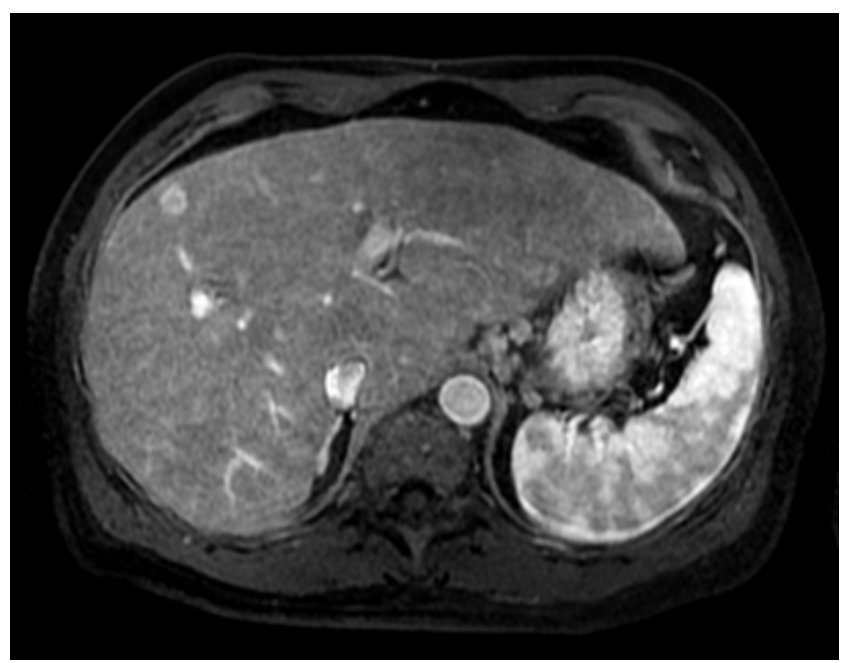

A

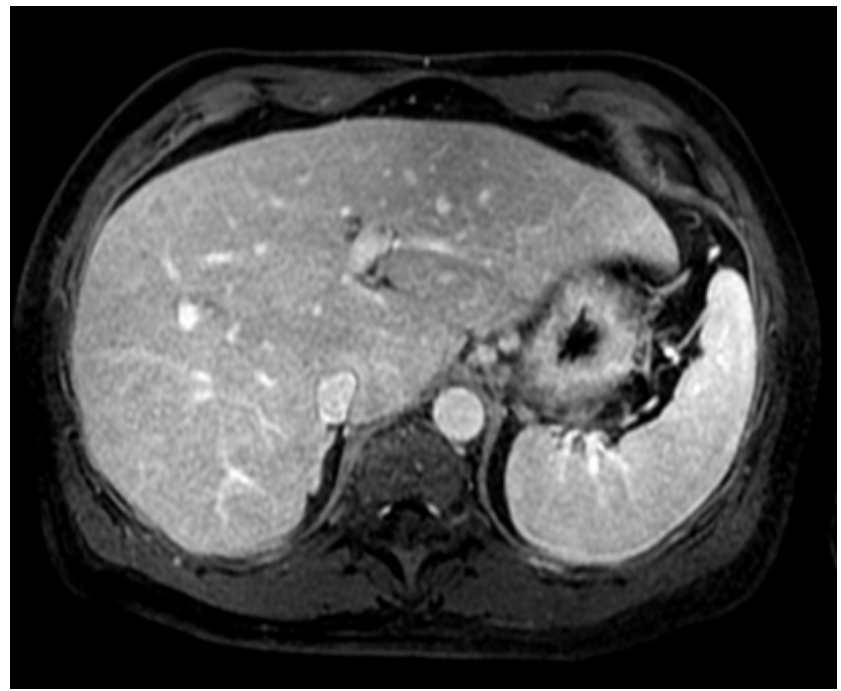

B

Figure 12. Fat saturated post-gadolinium $\mathrm{T} 1$ weighted gradient echo sequences in the late arterial (A) and delayed phase (B) demonstrating an enhancing mass with washout in the anterolateral aspect of the liver, corresponding to hepatocellular carcinoma. The presence of hyperenhancement relative to background liver is indicative of a solid mass with a vascular supply.

\section{Free and Bound Water}

Water exists in two main states in the human body: in an environment that allows for mobility (free water), and in an environment in which mobility is restricted (bound water). Many influences for bound water exist, such as impermeable membranes, impedance to movement by adjacent large macromolecules, and failure of the cell membrane sodium-potassium pump [13].

Free and bound water appears as bright on T2 weighted images. The higher the TE, or T2 weighting, the more a sequence is likely to display only free water. Modifying TE can control the degree of weighting. Moderate T2 weighting (TE $\sim 80 \mathrm{~ms}$ ) can be considered "water-sensitive" sequences, and heavy T2 weighting (TE $180 \mathrm{~ms}$ ) can be considered "water-specific sequences." In general, moderately or heavily weighted T2 sequences will both show freely mobile protons, but bound water will either be much less hyperintense than water or demonstrate no signal with increased T2 weighting (increased TE).

This concept can be used to differentiate lesions containing free water from bound water. For example, a cyst contains simple fluid that is freely mobile with few to no macromolecules, whereas hemangiomas contain fluid and relatively immobile macromolecules, such as proteins. While these two lesions are classically bright on T2 weighted sequences, the signal intensity of hemangiomas will be less than that of simple cysts as the TE increases (Figure 13), which can be used as a differentiating factor.

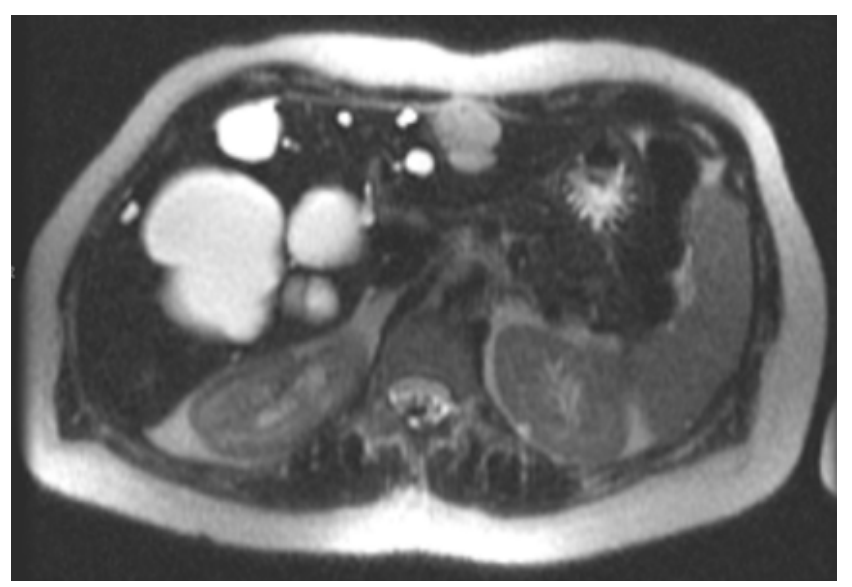

Figure 13. Moderately $\mathrm{T} 2$ weighted single shot sequence demonstrating the difference between free water and bound water. There are multiple cysts in the right hepatic lobe and medial segment left hepatic lobe which are extremely hyperintense due to signal from free water. Additionally, there is a small left hepatic lobe hemangioma which also demonstrates T2 hyperintensity which is less hyperintense than the cysts due to the presence of bound water.

The signal intensity of focal liver lesions relative to the spleen on T2 weighted sequences can also be used to differentiate malignant versus benign lesion. Lesions isointense to spleen are suspicious for malignancy; lesions hyperintense to spleen are usually benign such as cysts or hemangiomas [4].

Free water can also be identified on in and out of phase sequences. While conventionally used to identify microscopic fat, the converse can also be applied when 
imaging small amounts of water in the same voxel as macroscopic fat. In the setting of edema within peritoneal or retroperitoneal fat, inflammatory fluid and mesenteric or retroperitoneal fat will be located within the same voxel, leading to low signal on out of phase sequences, an added benefit of this sequence (Figure 14).

MRCP utilizes a very heavily weighted T2 sequence (TE $>500 \mathrm{~ms}$ ) such that only signal from simple fluid will contribute to image contrast and can be considered a "water only sequence." Simple cysts, the collecting system, CSF, and the biliary tree are common structures that contribute signal on this sequence due to their composition of mainly free water, allowing for an isolated view of the biliary tree (Figure 15).

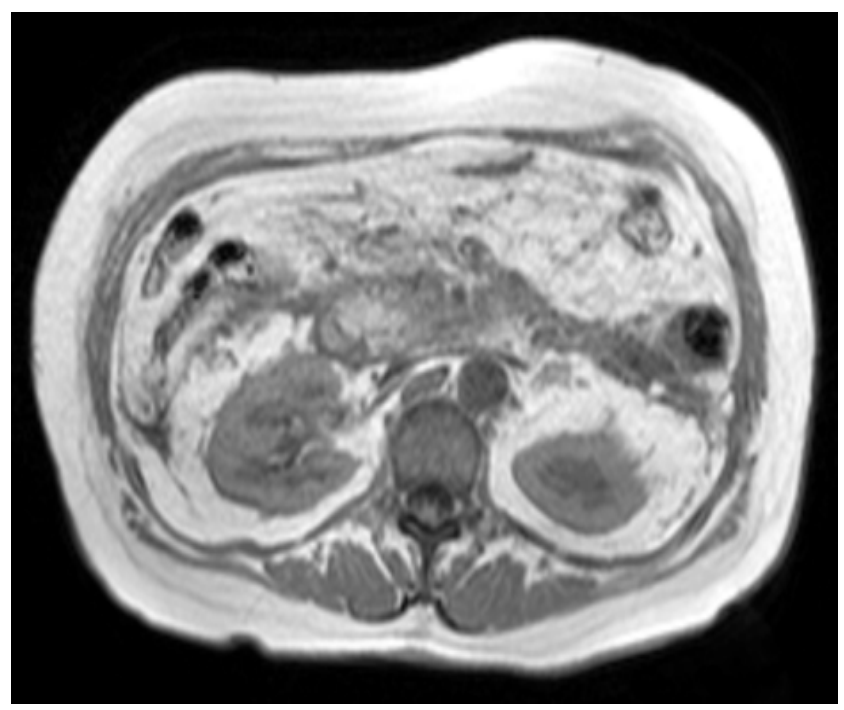

A

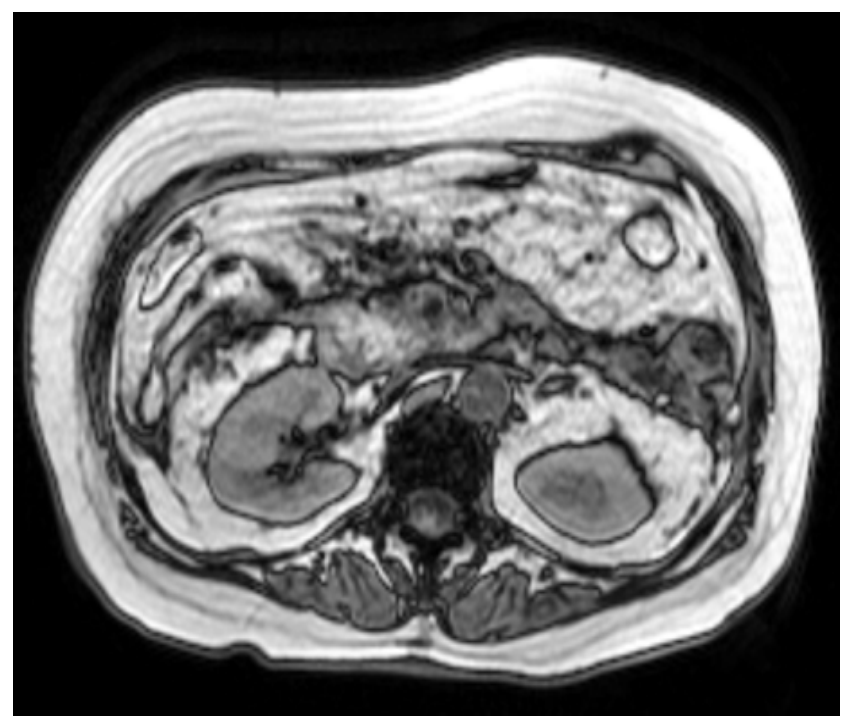

B

Figure 14A and 14B. In phase (A) and out of phase (B) gradient echo sequences demonstrating low signal intensity adjacent to a small bowel loop in the right midabdomen, which represents inflammatory fluid. Notice it remains dark on both sequences due to the presence of both fat and water within the same voxel.

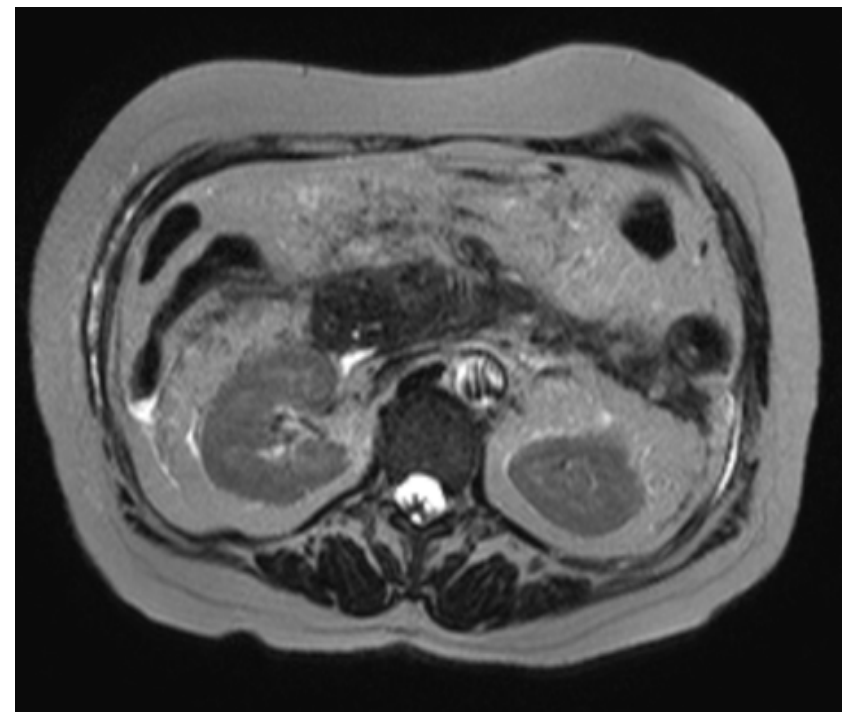

Figure 14C. Moderately weighted axial T2 weighted sequence confirming the presence of fluid as T2 hyperintensity adjacent to small bowel loop in the right midabdomen corresponding to the area of signal dropout on out of phase sequence (14B).

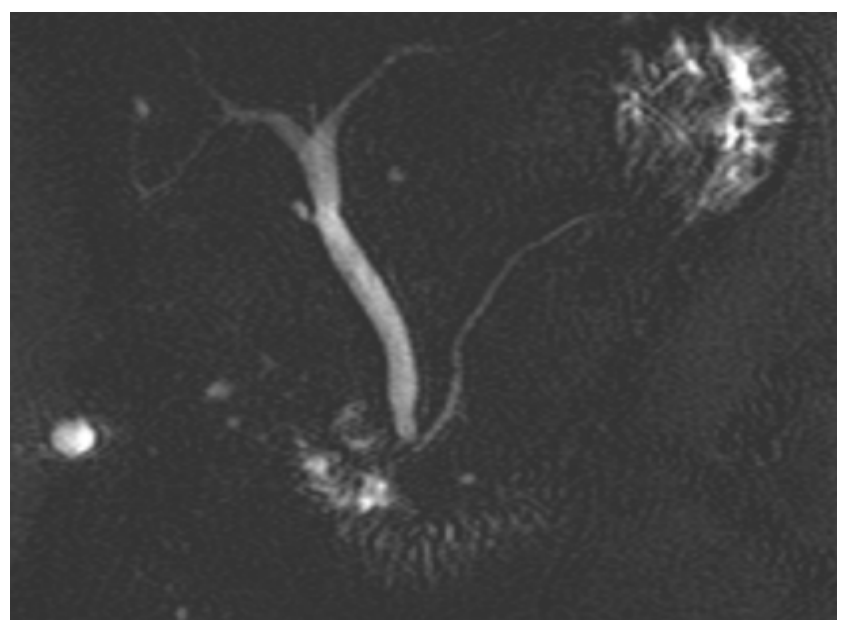

Figure 15. Heavily $\mathrm{T} 2$ weighted MRCP shows only fluid in the biliary tree, gastric and duodenal lumens. A right renal lesion visualized on the heavily T2 weighted sequence confirms a simple cyst identified on other sequences which are not shown here. Lesions with bound water cannot be visualized on heavily $\mathrm{T} 2$ weighted sequences.

\section{Conclusions}

The proliferation of non-intuitive acronyms and the inherent complexity of MR physics conspire to render abdominal MRI intimidatingly abstruse. Acknowledging the tissue-specific properties of each abdominal MRI pulse sequence can serve as the foundation to a rational approach to interpretation.

Tissue specific contrast can be applied to formulate an individual or institutional approach without changing protocols. While an understanding of the physics behind each sequence can be helpful, it serves as an adjunct to understanding why each sequence creates tissue contrast, rather than a means of clinical interpretation. 
Identification of a few key substances such as fats, paramagnetic substances, free or bound water, fibrous tissue, or protein is a universal approach that can be applied to any sequence. If the imager has an understanding for which particular substance a given sequence can identify, acronyms and names of sequences are much less important, and will become less of a burden to interpretation of abdominal MRI. As new sequences are brought into mainstream MR imaging, review of tissue specific imaging will continue to apply and will continue to gain relevance in regard to imaging interpretation.

\section{REFERENCES}

[1] A. Elster, J.H. Burdette. Questions \& Answers in Magnetic Resonance Imaging, 2nd Edition. St Louis, MO: Mosby, 2007, pp 32-42, 123-129.

[2] D.G. Mitchell, M. Crovello, T. Matteucci, R.O. Petersen, M.M. Miettinen. Benign adrenocortical masses: diagnosis with chemical shift MR imaging. Radiology 1992; 185:345-351.

[3] F. Fujiyoshi, M. Nakajo, Y. Fukukura, S. Tsuchimochi. Characterization of adrenal tumors by chemical shift fast low-angle shot MR imaging: comparison of four methods of quantitative evaluation. AJR 2003; 180:1649-1657

[4] E.S. Siegelman, A. Chauhan. MR Characterization of Focal Liver Lesions. Magn Reson Imaging Clin N Am. 2014; 22: 295-313.
[5] J.M. Virtanen, T.K. Pudas, J.A. Ratilainen, J.P. Saunavaara, M.E. Komu, R.K. Parkkola. Iron overload: accuracy of in-phase and out-of-phase MRI as a quick method to evaluate liver iron load in haematological malignancies and chronic liver disease. $\mathrm{Br} \mathrm{J}$ Radiol. 2012; 85(1014): e162-e167.

[6] F.F. Gugliemo, D.M. Mitchell, C.G. Roth, S. Deshmukh. Hepatic MR Imaging Techniques, Optimization, and Artifacts. Magn Reson Imaging Clin N Am. 2014; 22:263-282.

[7] S. Brandao, D. Seixas, M. Ayeres-Basto, S. Castro, J. Neto, C. Martins,, J.C. Ferreira, F. Parada. Comparing T1-weighted and T2-weighted three-point Dixon technique with conventional T1-weighted fat-saturation and short-tau inversion recovery techniques for the study of the lumbar spine in a short-bore MRI machine. Clin Radiol 2013; 68(11):e617-23.

[8] F.M. Saddiqui, S.V. Bekker, A.I. Quereshi. Neuroimaging of Hemorrhage and Vascular Defects. Neurotherapeutics 2011; 8(1): 28-38.

[9] D.T. Ginat, S.P. Meyers. Intracranial lesions with high signal intensity on T1-weighted MR images: differential diagnosis.Radiographics 2012; 32(2):499-516.

[10] C.G. Roth, S. Deshmukh. MRI of the pancreaticobiliary system. In: Fundamentals of body MRI. Philadelphia, PA: Elsevier Saunders; 2012: 129-199.

[11] F.H. Miller, A.L. Keppke, A. Wadhwa, J.N. Ly, K. Dalal, V.A. Kamler. MRI of Pancreatitis and Its Complications: Part 2, Chronic Pancreatitis. AJR 2004;183:1645-1652.

[12] J. Folkman. Role of angiogenesis in tumor growth and metastasis. Semin Oncol. 2002;29:15-18.

[13] S.H. Koenig. Molecular Basis of Magnetic Relaxation of Water Protons of Tissue. Acad Radiol 1996;3:597-606. 